\title{
MiR-193a-3p is an Important Tumour Suppressor in Lung Cancer and Directly Targets KRAS
}

\author{
Qian Fan ${ }^{a}$ Xiuting Hu ${ }^{b}$ Haiyang Zhang ${ }^{a}$ Shengguang Wang ${ }^{a}$ Huilai Zhang ${ }^{a}$ \\ Chaoying You $^{\mathrm{a}}$ Chen-Yu Zhang $^{\mathrm{b}}$ Hongwei Liang $^{\mathrm{b}}$ Xi Chen ${ }^{\mathrm{b}} \quad \mathrm{Yi} \mathrm{Ba}^{\mathrm{a}}$
}

aTianjin Medical University Cancer Institute and Hospital, National Clinical Research Center for Cancer, Key Laboratory of Cancer Prevention and Therapy, Tianjin, Tianjin's Clinical Research Center for Cancer, Tianjin, ${ }^{b}$ State Key Laboratory of Pharmaceutical Biotechnology, NJU Advanced Institute for Life Sciences, Jiangsu Engineering Research Center for MicroRNA Biology and Biotechnology, School of Life Sciences, Nanjing University, Nanjing, China

\section{Key Words}

MicroRNA $\cdot$ KRAS $・ N S C L C \cdot \operatorname{miR}-193 a-3 p$

\begin{abstract}
Background/Aims: MicroRNAs (miRNAs) have emerged as major regulators of tumour development and progression in non-small cell lung cancer (NSCLC). However, the role of miR-193a-3p in NSCLC is still unclear. Methods: Quantitative RT-PCR was used to detect miR-193a-3p expression levels in NSCLC tumour tissues. CCK8, EdU and cell migration assays were performed to analyse the biological functions of miR-193a-3p in NSCLC cells. Luciferase reporter assays were used to validate the bioinformatics-predicted target genes of miR193a-3p. Western blotting and RNA/DNA interference carried out to evaluate the association between miR-193a-3p and KRAS. Results: miR-193a-3p expression was decreased in the NSCLC tumour tissues. We investigated the biological effects of miR-193a-3p both in vivo and in vitro and found that enforced expression of miR-193a-3p inhibited tumour formation and suppressed cell proliferation and cell migration. KRAS was found to be a potential target of miR-193a-3p, and dual luciferase reporter assays showed that miR-193a-3p directly binds to the $3^{\prime}$-untranslated region (3'-UTR) of KRAS mRNA. In addition, we found that changing the expression of KRAS had the opposite results to those induced by miR-193a-3p in the NSCLC cells. Importantly, simultaneous overexpression of miR-193a-3p and KRAS could counteract the effects of both on cellular functions. Conclusion: These findings highlight an important role for miR-193a-3p as a tumour suppressor in NSCLC pathogenesis via the regulation of KRAS expression.
\end{abstract}




\section{Cellular Physiology Cell Physiol Biochem 2017;44:1311-1324 and Biochemistry Published online: November 29, 2017 www.karger.com/cpb \\ Fan et al.: MiR-193a-3p Inhibits KRAS in Lung Cancer}

\section{Introduction}

Lung cancer is the leading cause of cancer-related mortality worldwide. Non-small cell lung cancer (NSCLC) accounts for the majority (75-80\%) of lung cancer cases [1]. Although NSCLC has been investigated in numerous molecular studies aimed at developing new treatment strategies, the 5-year overall survival rate remains $<15 \%$ [2]. The exact mechanisms underlying oncogenesis and development of NSCLC remain complex and obscure, and thus, elucidation of the molecular basis of NSCLC and identification of new therapeutic targets for this disease are urgently needed.

MicroRNAs (miRNAs) are a class of endogenous, small, noncoding RNAs that are highly conserved across various species of animals and plants [3]. Since Calin et al. [4] first reported the role of miRNAs in cancer, many studies have confirmed the importance of miRNAs in carcinogenesis and cancer progression $[5,6]$. In mammals, miRNAs bind to complementary sites at the 3'-untranslated regions (3'-UTRs) of their targeted mRNAs, thereby inhibiting translation or favouring the destabilization of mRNAs, depending on the degree of nucleotide pairing. Anomalous miRNAs can exert a major effect by suppressing oncogenes or tumour suppressors, thereby functioning as tumour-suppressive miRNAs or oncogenic miRNAs during carcinogenesis. In past decade, the therapeutic potential of miRNAs in cancer has been demonstrated in an enormous number of published studies, including studies of NSCLC [7-9]. miR-193a, which consists of miR-193a-3p and miR-193a-5p, is one of the miRNAs involved in carcinogenesis. miR-193a-3p plays essential roles in the multi-chemoresistance of bladder cancer [10], epithelial ovarian cancer [11], breast cancer [12], colorectal cancer [13] and lung cancer [14]. However, although several studies indicating that miR-193a-3p is closely related to tumourigenesis have been published, the detailed roles of miR-193a-3p in the initiation and progression of NSCLC remain largely unknown. The aim of this study was to investigate the association between miR-193a-3p expression and NSCLC and to explore potential novel target genes of miR-193a-3p.

In this study, we demonstrated that miR-193a-3p levels were consistently downregulated in NSCLC tissues. We showed that miR-193a-3p inhibited tumour growth in an NSCLC cancer xenograft mouse model. Furthermore, we identified potential target genes of miR-193a-3p and found that miR-193a-3p might inhibit the viability, proliferation and migration of NSCLC cells by directly targeting a critical oncogene, KRAS.

\section{Materials and Methods}

\section{Human tissues and cell lines}

A total of 8 pairs of NSCLC tissues (LC) and matched noncancerous tissues (LN) were obtained from patients who underwent a surgical procedure at the Tianjin Medical University Cancer Institute and Hospital (Tianjin, China). The tissues were immediately frozen in liquid nitrogen and stored at $-80^{\circ} \mathrm{C}$. The clinical features of the NSCLC patients are listed in Table 1. The human NSCLC cell lines A549 and H1975 were purchased from the Shanghai Institute of Cell Biology, Chinese Academy of Sciences (Shanghai, China). The A549-Luc cells were produced by GenScript (Nanjing, China). All cells were cultured in Dulbecco's modified Eagle's medium (DMEM; GIBCO, Carlsbad, CA, USA) supplemented with 10\% foetal bovine serum (GIBCO). All cell lines were supplemented with $1 \%$ penicillin/streptomycin (Invitrogen, Carlsbad, CA, USA) and incubated in $5 \% \mathrm{CO}_{2}$ at $37^{\circ} \mathrm{C}$ in a water-saturated atmosphere.

\section{Establishment of tumour xenografts in mice}

BALB/c athymic male nude mice (nu/nu; each 5-6 weeks old and 16-20 g) were purchased from the Model Animal Research Centre of Nanjing University. All animal studies were carried out in accordance with the Guide for Animal Care and Use Committee at Nanjing University. The A549-Luc cells $\left(2 \times 10^{6}\right.$ cells $)$ suspended in $100 \mu \mathrm{l}$ of Dulbecco's PBS were injected into the flanks of nude mice. After 14 days, the transplanted nude mice were randomly divided into three groups ( $\mathrm{n}=5$ each group), and tumour volume was assessed by BLI. PBS, Chol-miR-NC or Chol-miR-193a-3p (RiboBio Co., Ltd., Guangzhou, China) was 


\section{Cellular Physiology Cell Physiol Biochem 2017;44:1311-1324 \begin{tabular}{ll|l} 
and Biochemistry $10.1159 / 000485491$ & $\begin{array}{l}\text { @ } 2017 \text { The Author(s). Published by S. Karger AG, Basel } \\
\text { www.karger.com/cpb }\end{array}$ \\
\hline
\end{tabular} Fan et al.: MiR-193a-3p Inhibits KRAS in Lung Cancer}

directly injected into the implanted tumour at a dose of $1 \mathrm{nmol}$ (in $20 \mu \mathrm{l}$ of phosphate-buffered saline) per mouse every other day for a total of 6 doses. Tumour volumes were assessed by BLI on days 14 and 28. The luminescence index from the 5 mice in each group were averaged. On day 28, the mice were euthanized. The tumour xenografts were removed, parts of the tumours were used for protein and total RNA extraction, and the remaining tumours were fixed in $4 \%$ paraformaldehyde for $24 \mathrm{~h}$ and then further processed for H\&E staining and immunohistochemical staining for Ki-67.

Table 1. Patients' Characteristics

\begin{tabular}{|c|c|c|c|c|c|c|c|}
\hline Case No & Age & Gender & Pathological Stage & Pathological type & EGFR & KRAS & ALK \\
\hline $1 \#$ & 55 & $\mathrm{M}$ & II & adenocarcinoma & Mut & $\mathrm{Wt}$ & - \\
\hline $2 \#$ & 42 & $\mathrm{~F}$ & I & adenocarcinoma & Mut & Wt & - \\
\hline $3 \#$ & 65 & M & II & adenocarcinoma & Wt & Wt & + \\
\hline 4\# & 48 & M & II & adenocarcinoma & Wt & Mut & - \\
\hline $5 \#$ & 57 & $\mathrm{~F}$ & II & $\begin{array}{l}\text { squamous cell } \\
\text { carcinoma }\end{array}$ & Wt & Wt & - \\
\hline $6 \#$ & 61 & M & III & adenocarcinoma & Wt & Wt & - \\
\hline 7\# & 56 & $\mathrm{~F}$ & II & adenocarcinoma & Mut & Wt & - \\
\hline $8 \#$ & 39 & $\mathrm{~F}$ & III & $\begin{array}{l}\text { squamous cell } \\
\text { carcinoma }\end{array}$ & Mut & Wt & - \\
\hline
\end{tabular}

miR-193a-3p overexpression or knockdown

miRNA overexpression was achieved by transfecting cells with a pre-miR-193a-3p, whereas knockdown was achieved by transfecting cells with an anti-miR-193a-3p. Synthetic pre-miR-193a-3p, anti-miR-193a3p, pre-miR-control and anti-miR-control RNAs were purchased from GenePharma (Shanghai, China). Cells were seeded in 6-well plates and were transfected using Lipofectamine 3000 (Invitrogen) according to the manufacturer's guidelines. In each well, 100 pmol pre-miR-193a-3p, anti-miR-193a-3p, or scrambled negative control RNA was used. The cells were harvested $24 \mathrm{~h}$ after transfection for quantitative RT-PCR and harvested $48 \mathrm{~h}$ after transfection for Western blotting.

RNA isolation and quantitative RT-PCR

Total RNA was isolated according to the manufacturer's instructions using TRIzol reagent (Invitrogen). Assays to quantify mature miRNAs were performed using TaqMan miRNA probes (Applied Biosystems, Foster City, CA, USA) according to the manufacturer's instructions. Quantitative RT-PCR was performed to quantify miRNA levels and mRNA levels (see our previous study for details [15]). The primers for PCR amplifications were as follows: KRAS (sense) 5'-GACTCTGAAGATGTACCTATGGTCCTA-3'; KRAS (antisense) 5'-CATCATCAACACCCTGTCTTGTC-3'; GAPDH (sense) 5'-GATATTGTTGCCATCAATGAC-3'; GAPDH (antisense) 5'-TTGATTTTGGAGGGATCTCG-3'. GAPDH and U6 were used as internal reference genes for mRNA and miRNA quantifications, respectively.

\section{Cell viability assay}

For determination of cell viability, $6 \mathrm{~h}$ after transfection, A549 and H1975 cells were seeded in triplicate in 96-well plates at a density of $1 \times 10^{4}$ cells/well in $100 \mu \mathrm{l}$ of culture medium. The cell proliferation index was measured using a Cell Counting Kit-8 (CK04-500, Dojindo, Japan) at 12, 24, 36, 48 and $60 \mathrm{~h}$ after transfection according to the manufacturer's instructions.

\section{EdU proliferation assay}

A549 cells were seeded into 48-well plates to assess cell proliferation. The cells were incubated under standard conditions in complete media (DMEM supplemented with 10\% FBS). Transfection of the cells and cell harvesting were performed the following day as described above. Cell proliferation was detected by the incorporation of 5-ethynyl-2'-deoxyuridine (EdU) using an EdU Cell Proliferation Assay Kit (RiboBio, Guangzhou, China). Briefly, the cells were incubated with $50 \mu \mathrm{M}$ EdU for $4 \mathrm{~h}$ before fixation, permeabilization 


\section{Cellular Physiology Cell Physiol Biochem 2017;44:1311-1324 \begin{tabular}{ll|l} 
and Biochemistry $10.1159 / 000485491$ & Published online: November 29, 2017 & $\begin{array}{l}\text { 2 2017 The Author(s). Published by S. Karger AG, Basel } \\
\text { ww.karger.com/cpb }\end{array}$ \\
\hline
\end{tabular} \\ Fan et al.: MiR-193a-3p Inhibits KRAS in Lung Cancer}

and EdU staining, which were performed according to the manufacturer's protocol. The cell nuclei were stained with Hoechst (RiboBio, Guangzhou, China). The proportion of nucleated cells incorporating EdU was determined by fluorescence microscopy.

\section{Cell migration assay}

The migration of A549 and H1975 cells were tested using a two-chamber Transwell cell migration assay (BD Biosciences, MA, USA), following the manufacturer's instructions. The cells were harvested $24 \mathrm{~h}$ after transfection, suspended in FBS-free DMEM culture medium and added to the upper chamber $\left(4 \times 10^{4}\right.$ cells per well). At the same time, $0.5 \mathrm{ml}$ of DMEM with $20 \%$ FBS was added to the lower compartment. The cells were allowed to migrate for $16 \mathrm{~h}$ in a $5 \% \mathrm{CO}_{2}$ atmosphere that was saturated with $\mathrm{H}_{2} \mathrm{O}$ at $37^{\circ} \mathrm{C}$. After incubation, cells in the upper surface of the Transwell chamber were removed using cotton swabs. Migrated cells attached on the undersurface were fixed with 4\% paraformaldehyde for $10 \mathrm{~min}$ and stained with crystal violet solution $(0.5 \%$ in methanol) for $15 \mathrm{~min}$ at room temperature. The lower surfaces (with cells that had migrated) were imaged using a photomicroscope $(10 \times$ fields per chamber) (BX51, Olympus, Japan).

\section{miRNA target prediction}

Genes that may be targeted by miR-193a-3p were determined using three computational algorithms, including TargetScan (http://genes.mit.edu/targetscan/), PicTar (http://pictar.bio.nyu.edu/) and miRanda (http://cbio.mskcc.org/cgi-bin/mirnaviewer/mirnaviewer.pl).

\section{Luciferase reporter assay}

For analysis of the direct binding of miR-193a-3p to the target gene KRAS, a luciferase reporter assay was performed as previously described [16]. The entire 3'-UTR of human KRAS was cloned and inserted downstream of a p-MIR-reporter plasmid (Ambion). For assessment of the binding specificity, the sequences that interacted with the miR-193a-3p seed sequence were mutated (both binding positions were mutated).

\section{siRNA and plasmid construction interference assay}

The siRNA sequence targeting human KRAS CDNA was designed and synthesized by GenePharma (Shanghai, China). The siRNA sequence was 5'-GGAAGCAAGUAGUAAUUGATT-3'. The scrambled siRNA was included as a negative control. A mammalian expression plasmid (pReceiver-M02-KRAS) was purchased from GeneCopoeia (Germantown, MD, USA). An empty plasmid (pReceiver-M02) served as a negative control. The KRAS siRNA or KRAS overexpression plasmid was transfected into A549 and H1975 cells using Lipofectamine 3000 (Invitrogen) according to the manufacturer's instructions. Total RNA or protein was isolated 24 or $48 \mathrm{~h}$ after transfection. Quantitative RT-PCR and Western blotting were used to assess the KRAS mRNA and protein levels, respectively.

\section{Protein isolation and Western blotting}

All of the cells were rinsed with PBS (pH 7.4) and lysed in RIPA Lysis buffer (Beyotime, China) supplemented with a protease and phosphatase inhibitor cocktail (Thermo Scientific 78440) on ice for 45 min. The tissue samples were frozen with liquid nitrogen, ground into a powder and lysed in RIPA Lysis buffer containing the protease and phosphatase inhibitor cocktail on ice for $60 \mathrm{~min}$. When necessary, sonication was used to facilitate cell lysis. Cell lysates or tissue homogenates were centrifuged for $15 \mathrm{~min}$ $\left(12,000 \mathrm{~g}, 4^{\circ} \mathrm{C}\right)$. The supernatant was collected, and the protein concentration was calculated using a Pierce BCA protein assay kit (Thermo Scientific, Rockford, IL, USA). The protein levels were analysed using Western blotting with the corresponding antibodies. Densitometry analysis was performed using ImageJ and normalized by the GAPDH intensity.

\section{Statistical analysis}

All Western blotting images are representative of at least three independent experiments. Quantitative RT-PCR and the luciferase reporter, cell viability, cell proliferation and cell migration assays were performed in triplicate, and each experiment was repeated at least 3 times. Data (mean \pm SEM) are representative of at least three independent experiments. The numerical data were statistically analysed by two-tailed Student's t-test. Statistically significance was defined as $\mathrm{P}<0.05$. 


\section{Cellular Physiology Cell Physiol Biochem 2017;44:1311-1324

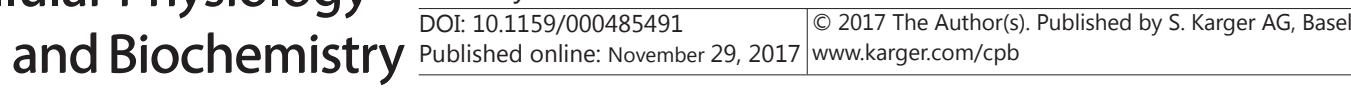 Fan et al.: MiR-193a-3p Inhibits KRAS in Lung Cancer}

\section{Results}

miR-193a-3p is downregulated in NSCLC tissues

We assessed the expression patterns of miR-193a-3p in 8 NSCLC tissues and 8 normal adjacent tissues using quantitative RT-PCR. Consistent with previous studies, miR-193a-3p levels were found to be consistently downregulated in NSCLC tissues compared to normal adjacent tissues (Fig. 1).

miR-193a-3p functions as a tumour suppressor in NSCLC

Then, we evaluated the biological effects of miR-193a-3p on NSCLC tumourigenesis in a xenograft mouse model. A549Luc cells were subcutaneously implanted into 6-week-old SCID mice; after 14 days, the transplanted nude mice were randomly divided into three groups ( $\mathrm{n}=5$ each group). PBS ( $\mathrm{pH} 7.4$ ), Chol-miR-NC or Chol-miR-193a$3 p$ was directly injected into the implanted tumour every other day for a total of six doses, and tumour volumes were assessed by BLI. The luminescence index from the 5 mice in each group was averaged. The fold increase in BLI was calculated as the proportion of post-treatment BLI (28 days) to baseline BLI (14 days) (Fig. 2A). A significant suppression in the photon count of the tumours was observed in the Chol-miR-193a-3p-treated group compared to the PBS or Chol-miR-NC-treated groups (Fig. 2B). Subsequently, total RNA was extracted from each xenograft and used to evaluate the expression of miR-193a-3p. After two weeks of intratumoural treatment, tumours from the Chol-miR-193a-3p-treated group showed a significant increase in miR-193a-3p expression compared to tumours from the PBS- or CholmiR-NC-treated groups (Fig. 2C). Furthermore, tumour tissues were embedded in paraffin and then stained with haematoxylin and eosin (H\&E) for histological examination. The results revealed less cell mitosis in the Chol-miR-193a-3p-treated group than in the PBS- or Chol-miR-NC-treated groups (Fig. 2D). Finally, the proliferative activity of the tumour cells was assessed via immunohistochemical staining of Ki-67. The tumour cell proliferation rate, as measured by the staining intensity of Ki-67-positive cells, was reduced in tumours from the Chol-miR-193a-3p-treated group (Fig. 2E and 2F).

\section{Effect of miR-193a-3p in the regulation of proliferation, viability and migration of NSCLC cells}

We investigated whether miR-193a-3p overexpression or knockdown would impact the functions of NSCLC cells. In these experiments, miR-193a-3p was overexpressed by more than 100-fold following transfection of the A549 or H1975 NSCLC cell lines with premiR-193a-3p, and miR-193a-3p knockdown reduced expression by approximately 50\% after transfection of A549 and H1975 cells with anti-miR-193a-3p. The efficiency of the overexpression or knockdown of miR-193a-3p in A549 and H1975 cells is shown in (Fig. 3A). The proliferation of A549 cells, as measured by the percentage of EdU-positive cells in the EdU assay, was decreased approximately $40 \%$ in A549 cells transfected with pre-miR-193a$3 p$; by contrast, knockdown of miR-193a-3p had the opposite effect on A549 cell proliferation (Fig. 3B and 3C). Moreover, cell viability was investigated via CCK8 assays. Overexpression of miR-193a-3p suppressed the viability of A549 and H1975 cells, while inhibition of miR193a-3p increased cell viability (Fig. 3D and 3E). Furthermore, we analysed the effects of miR-193a-3p on the migration of A549 and H1975 cells using Transwell assays. As expected, 


\section{Cellular Physiology Cell Physiol Biochem 2017;44:1311-1324 \begin{tabular}{ll|l|l|l|l} 
DO 2017 The Author(s). Published by S. Karger AG, Basel \\
and Biochemistry
\end{tabular} \\ Fan et al.: MiR-193a-3p Inhibits KRAS in Lung Cancer}

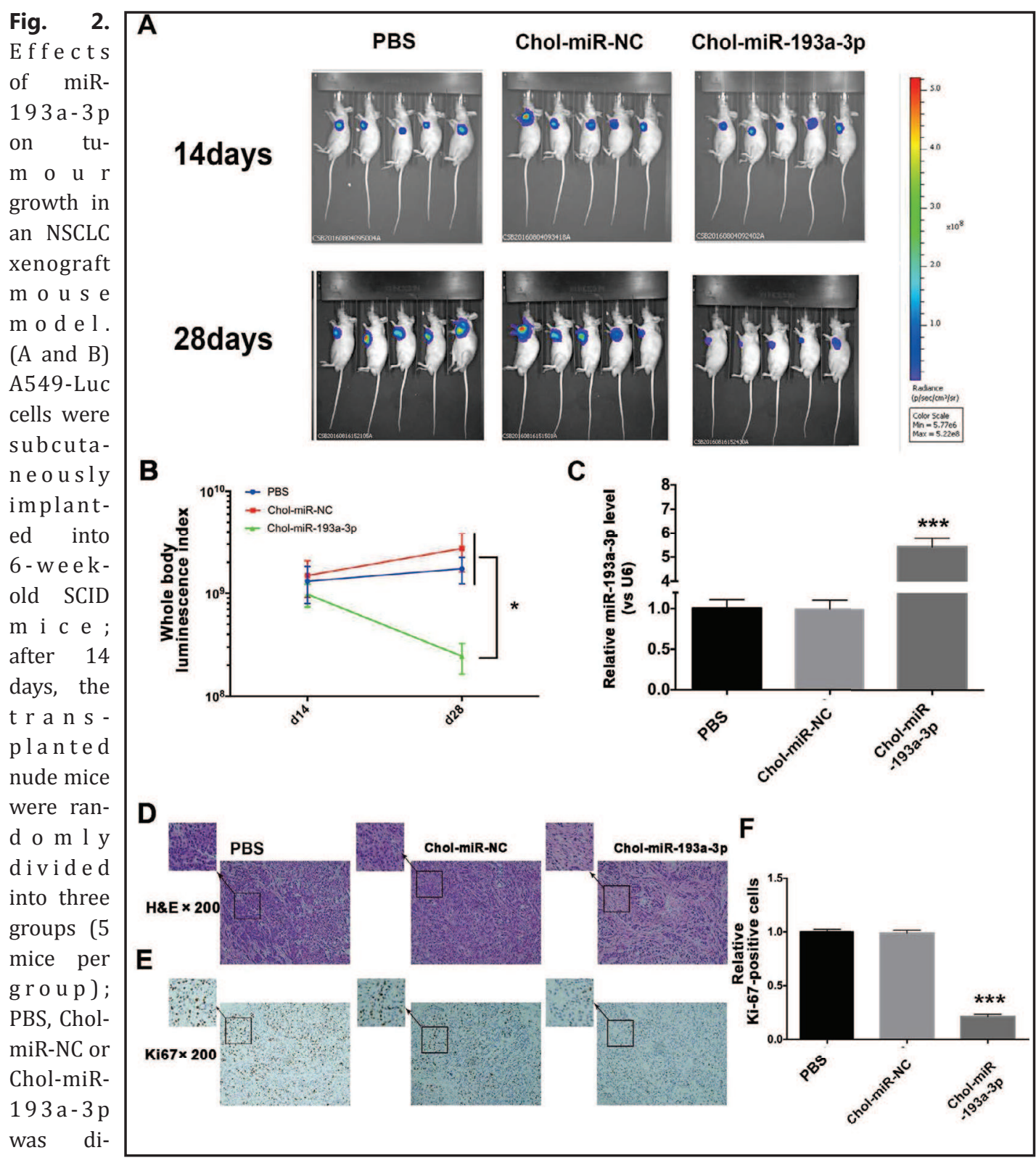

rectly in-

jected into the implanted tumour every other day for a total of six doses. Tumour volumes were assessed by BLI on days 14 and 28. The intensity of BLI is represented by the colour. (A) A representative image. (B) Photon counts of mice from each group were averaged. (C) Quantitative RT-PCR analysis of miR-193a-3p levels in tumours from mice treated with PBS, Chol-miR-NC or Chol-miR-193a-3p. (D and E) The tumours from mice treated with PBS, Chol-miR-NC or Chol-miR-193a-3p were subjected to H\&E staining and immunohistochemical staining for Ki-67. (F) Quantitative analysis of Ki-67. ${ }^{*} \mathrm{P}<0.05,{ }^{* *} \mathrm{P}<0.01,{ }^{* * *} \mathrm{P}<0.001$.

cells transfected with pre-miR-193a-3p showed a reduction in cell motility, whereas the cells transfected with anti-miR-193a-3p showed enhanced cell motility (Fig. 3F and 3G).

Prediction of KRAS as a target gene of miR-193a-3p

To explore the molecular mechanism by which miR-193a-3p contributes to the tumour suppression of NSCLC, we used three publicly available algorithms, including TargetScan [17], PicTar [18] and miRanda [19], in combination to search for potential targets of miR- 


\section{Cellular Physiology Cell Physiol Biochem 2017;44:1311-1324 \begin{tabular}{ll|l} 
and Biochemistry & Dublished online: November 29, 2017 & $\begin{array}{l}\text { @ } 2017 \text { The Author(s). Published by S. Karger AG, Basel } \\
\text { www.karger.com/cpb }\end{array}$ \\
\hline
\end{tabular} Fan et al.: MiR-193a-3p Inhibits KRAS in Lung Cancer}

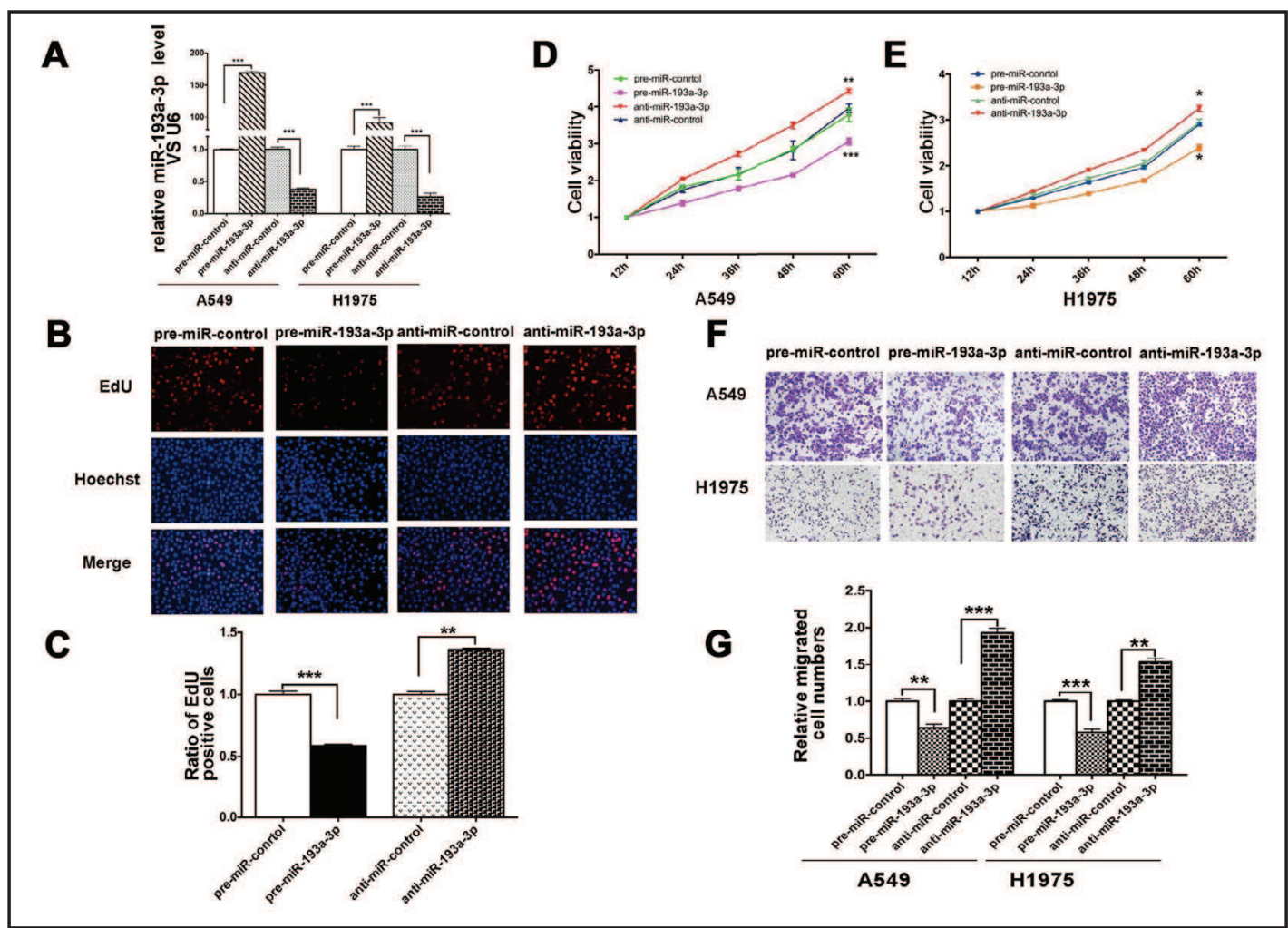

Fig. 3. Effect of miR-193a-3p in the regulation of viability, proliferation and migration of lung cancer cells. (A) Quantitative RT-PCR analysis of miR-193a-3p levels in A549 and H1975 cells transfected with equal doses A549 and H1975 of pre-miR-193a-3p, anti-miR-193a-3p or scrambled negative control RNAs (premiR-control or anti-miR-control). (B and C) The EdU assay of proliferation. A549 cells were transfected with equal doses of pre-miR-control, pre-miR-193a-3p, anti-miR-control or anti-miR-193a-3p; B, representative image; C, quantitative analysis. (D and E) CCK8 viability assays were performed 12, 24, 36, 48 and $60 \mathrm{~h}$ after the transfection of A549 or H1975 cells with pre-miR-control, pre-miR-193a-3p, anti-miR-control or antimiR-193a-3p. (F and G) Transwell analysis of the migration rate of A549 or H1975 cells treated with equal doses of pre-miR-control, pre-miR-193a-3p, anti-miR-control or anti-miR-193a-3p; F, representative image; $\mathrm{G}$, quantitative analysis. ${ }^{* *} \mathrm{P}<0.01,{ }^{* * *} \mathrm{P}<0.001$.

193a-3p. Among the candidates, KRAS, an oncogene that is frequently upregulated in NSCLC, was predicted to be a miR-193a-3p target by all three of the algorithms and was selected for further experimental verification. The predicted interaction between miR-193a-3p and the target site in the KRAS 3'-UTR is shown in Fig. 4A. Two predicted hybridizations were observed between miR-193a-3p and the 3'-UTR of KRAS. The minimum free energy values of the two hybridizations between miR-193a-3p and KRAS are -16.7 and $-22.4 \mathrm{kcal} / \mathrm{mol}$, which are well within the range of genuine miRNA-target pairs.

Theoretically, miRNAs are believed to have expression patterns that are opposite to those of their targets [20,21]. We next investigated whether miR-193a-3p was inversely correlated with KRAS in NSCLC tissues. We measured the expression pattern of KRAS in the same 8 pairs of NSCLC tissues and normal adjacent tissues. KRAS protein levels were consistently increased in NSCLC tissues (Fig. 4B and 4C). In contrast, KRAS mRNA levels did not significantly differ between the cancerous and noncancerous tissues (Fig. 4D), which is consistent with the involvement of a post-transcriptional mechanism in the regulation of KRAS. The inverse correlation between miR-193a-3p and KRAS protein levels (Fig. 4E) and the disparity between the miR-193a-3p and KRAS mRNA levels (Fig. 4F) were further illustrated using Pearson's correlation scatter plots. Moreover, we found similar results in tumours from the xenograft mice. Tumours from the Chol-miR-193a-3p-treated group 

Cellular Physiology Cell Physiol Biochem 2017;44:1311-1324 \begin{tabular}{l|l|l} 
DOI: 10.1159/000485491 & (c) 2017 The Author(s). Published by S. Karger AG, Basel
\end{tabular}

Fig. 4. KRAS

was predicted as a target of miR-193a-3p and was upregulated in lung cancer tissues. (A) Schematic description of the hypothetical duplexes formed by the interaction between the binding sites in the KRAS 3'-UTR (top) and miR-193a-3p (bottom). The predicted free energy value of each hybrid is indicated. The seed sequences and seed recognition sites are indicated in red, and all nucleotides in these regions are highly conserved in several species. (B and C) Western

blotting analysis of KRAS protein levels in 8 pairs of LC and LN samples; B, representative image; C, quantitative analysis. (D) Quantitative RT-PCR analysis of KRAS mRNA levels in the same 8 pairs of LC and LN samples. (E and F) Pearson's correlation scatter plot of the fold change in the miR-193a-3p and KRAS protein levels in the same 8 pairs of LC and LN samples. ( $G$ and H) Western blotting analysis of KRAS protein or KRAS mRNA levels in tumours from mice treated with PBS, Chol-miR-NC or Chol-miR-193a-3p; G, representative image; $\mathrm{H}$, quantitative analysis. ${ }^{*} \mathrm{P}<0.05,{ }^{*} \mathrm{P}<0.01$.

displayed reduced KRAS protein levels (Fig. 4G and 4H), but not mRNA levels, compared to tumours from the control groups. Thus, KRAS was identified as a target of miR-193a-3p based on both computational predictions and the inverse correlation between miR-193a-3p and KRAS protein levels, but not mRNA levels, in NSCLC tissues.

Validation of KRAS as a direct target of miR-193a-3p

The correlation between miR-193a-3p and KRAS expression was further investigated by evaluating KRAS expression in A549 and H1975 NSCLC cell lines after overexpression or knockdown of miR-193a-3p. The expression of KRAS protein was reduced approximately $50 \%$ by miR-193a-3p overexpression and increased by miR-193a-3p knockdown in A549 and H1975 cells (Fig. 5A and 5B). To determine the level at which miR-193a-3p regulates KRAS expression, we repeated the above experiments and examined the expression of KRAS mRNA after transfection. Overexpression or knockdown of miR-193a-3p did not affect the 


\section{Cellular Physiology Cell Physiol Biochem 2017;44:1311-1324

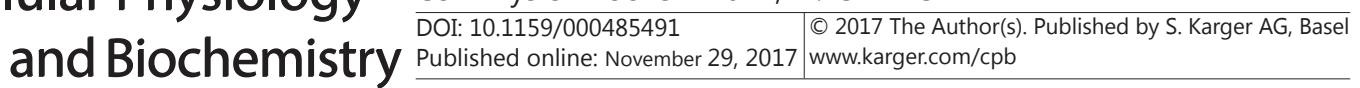 \\ Fan et al.: MiR-193a-3p Inhibits KRAS in Lung Cancer}

Fig. 5. KRAS was a direct target of miR-193a-3p. (A and B) Western blotting analysis of KRAS protein levels in A549 and H1975 cells transfected with equal doses of the pre-miR193a-3p, antimiR - 193 a-3 p or scrambled negative control RNAs; A, representative image; B, quantitative analysis. (C) Quantitative RT-PCR analysis of KRAS mRNA levels in A549 and H1975 cells transfected with equal doses of pre-miR193a-3p, antimiR-193a-3p or scrambled negative control RNAs. (D) Western blotting analysis of the phosphorylation levels of MEK, MAPK and YAP1 in A549 cells were transfected with equal doses of pre-miR-con-
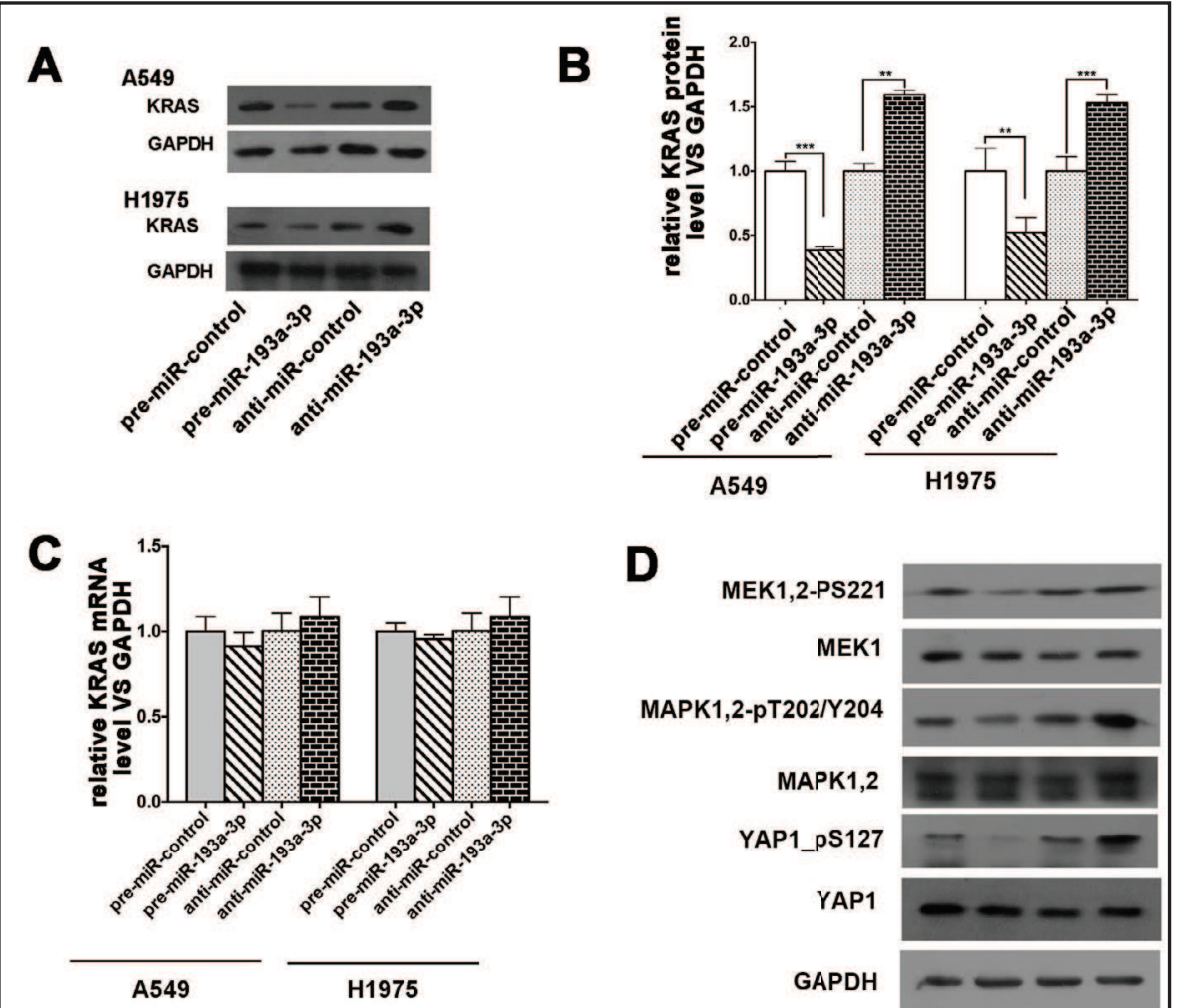

MAPK1,2-pT202/Y204

MAPK1,2

YAP1_pS127

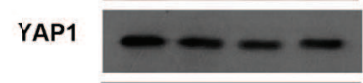

E

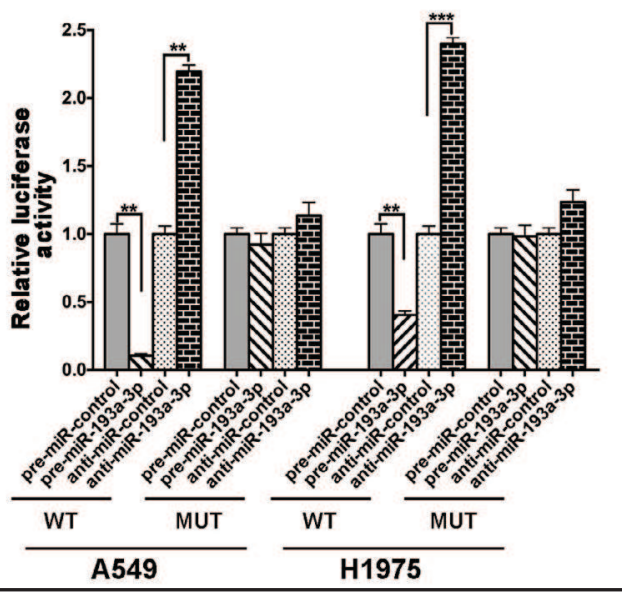

GAPDH

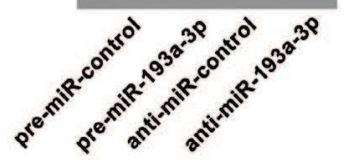

trol, pre-miR-

193a-3p, anti-miR-control or anti-miR-193a-3p. (E) Firefly luciferase reporters containing wild-type (WT) or mutant (MUT) miR-193a-3p binding sites in the KRAS 3'-UTR were co-transfected into A549 and H1975 cells along with pre-miR-control, pre-miR-193a-3p, anti-miR-control or anti-miR-193a-3p. Twenty-four hours post-transfection, the cells were assayed using a luciferase assay kit. ${ }^{* *} \mathrm{P}<0.01,{ }^{* * *} \mathrm{P}<0.001$.

mRNA levels of KRAS (Fig. 5C). These results demonstrated that miR-193a-3p specifically regulated KRAS protein expression at the post-transcriptional level, which is the most common mechanism of animal miRNA action. To verify that the downstream KRAS pathway inversely correlated with miR-193a-3p, we overexpressed/knocked down miR-193a-3p in the A549 cell line. The phosphorylation levels of MEK, MAPK and YAP1 were all reduced upon expression of miR-193a-3p (Fig. 5D). 


\section{Cellular Physiology Cell Physiol Biochem 2017:44:1311-1324 \begin{tabular}{l|l|l} 
DOI: 10.1159/000485491 & ( 2017 The Author(s). Published by S. Karger AG, Basel
\end{tabular}

Fig. 6. Effect of KRAS in the regulation of viability, proliferation and migration of lung cancer cells. (A and B) Western blotting analysis of KRAS protein levels in A549 cells transfected with equal doses of control siRNA or KRAS siRNA or with equal doses of control vector or KRAS vector; A, representative image; $\mathrm{B}$, quantitative analysis. (C) Quantitative RT-PCR analysis of KRAS mRNA levels in A549 cells transfected with equal doses of control siRNA or KRAS siRNA or with equal doses of control vector or KRAS vector. (D) CCK8 viability assays were performed 12, $24,36,48$ and $60 \mathrm{~h}$ after the transfection of A549 cells with control SiRNA or KRAS siRNA $r$ with equal doses of control vector or KRAS vector. ( $E$ and F) EdU assay of proliferation of A549 cells transfected with equal doses of control siRNA or KRAS siRNA or with equal doses of control vector or KRAS vector; E, rep-
A

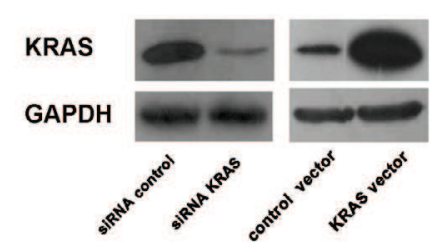

C

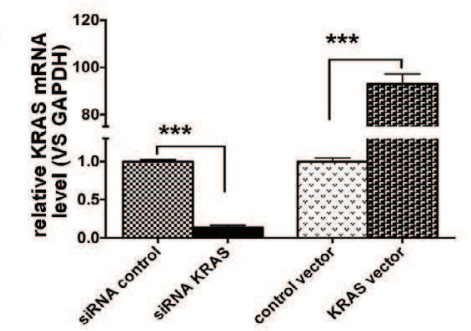

E

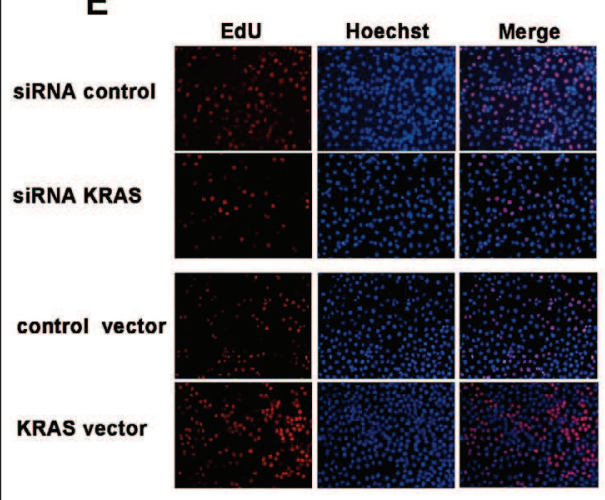

G

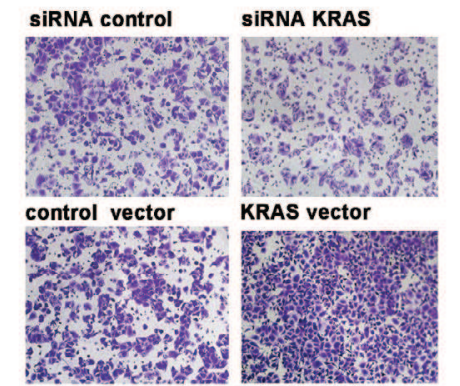

B

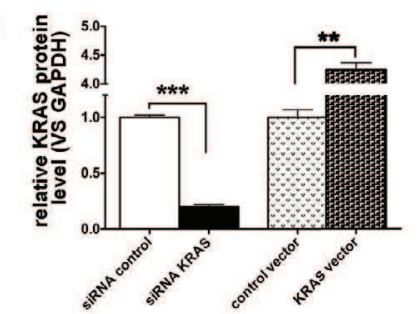

D

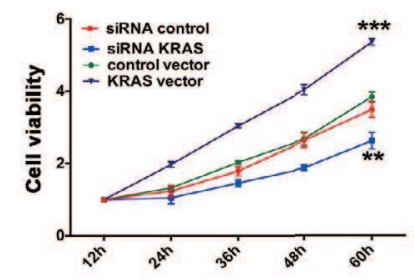

$\mathbf{F}$

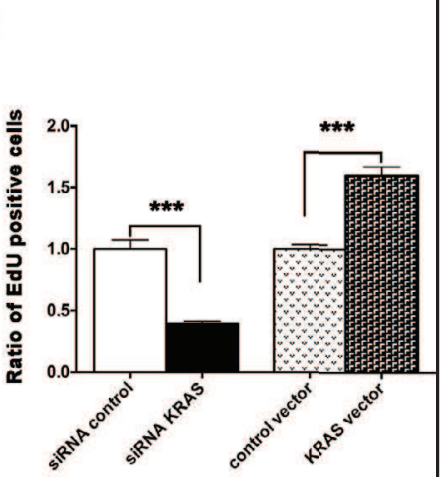

H

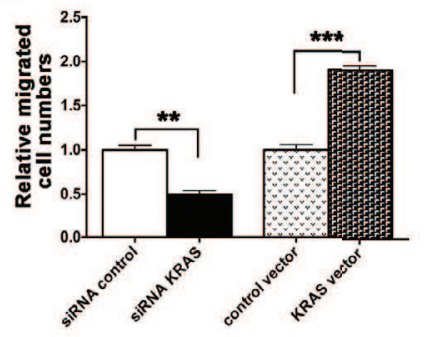

resentative image; F, quantitative analysis. ( $G$ and $H$ ) Transwell analysis of migration of A549 cells treated with equal doses of control siRNA or KRAS siRNA or with equal doses of control vector or KRAS vector; G, representative image; $\mathrm{H}$, quantitative analysis. ${ }^{* *} \mathrm{P}<0.01,{ }^{* * *} \mathrm{P}<0.001$.

To confirm that miR-193a-3p directly targets the presumed binding sites in the KRAS 3'UTR, negatively regulating KRAS expression, we performed luciferase reporter assays. The KRAS 3'-UTR containing the presumed miR-193a-3p binding sites was fused downstream of the firefly luciferase gene in a reporter plasmid. The recombinant plasmid was transfected into A549 and H1975 cells along with pre-miR-193a-3p or anti-miR-193a-3p. As expected, the luciferase activity was reduced in the cells transfected with pre-miR-193a-3p, whereas inhibition of miR-193a-3p resulted in an increase in reporter activity compared with transfection with the anti-miR-control. Furthermore, we introduced point mutations into the corresponding complementary sites in the 3'-UTR of KRAS to disrupt the predicted miR-193a-3p binding sites (both binding positions were mutated). This mutated luciferase 


\section{Cellular Physiology Cell Physiol Biochem 2017;44:1311-1324 and Biochemistry Published online: November 29, $2017 \mid \begin{aligned} & \text { @ } 2017 \text { The Author(s). Published by S. Karger AG, Basel } \\ & \text { wwrger.com/cpb }\end{aligned}$ \\ Fan et al.: MiR-193a-3p Inhibits KRAS in Lung Cancer}

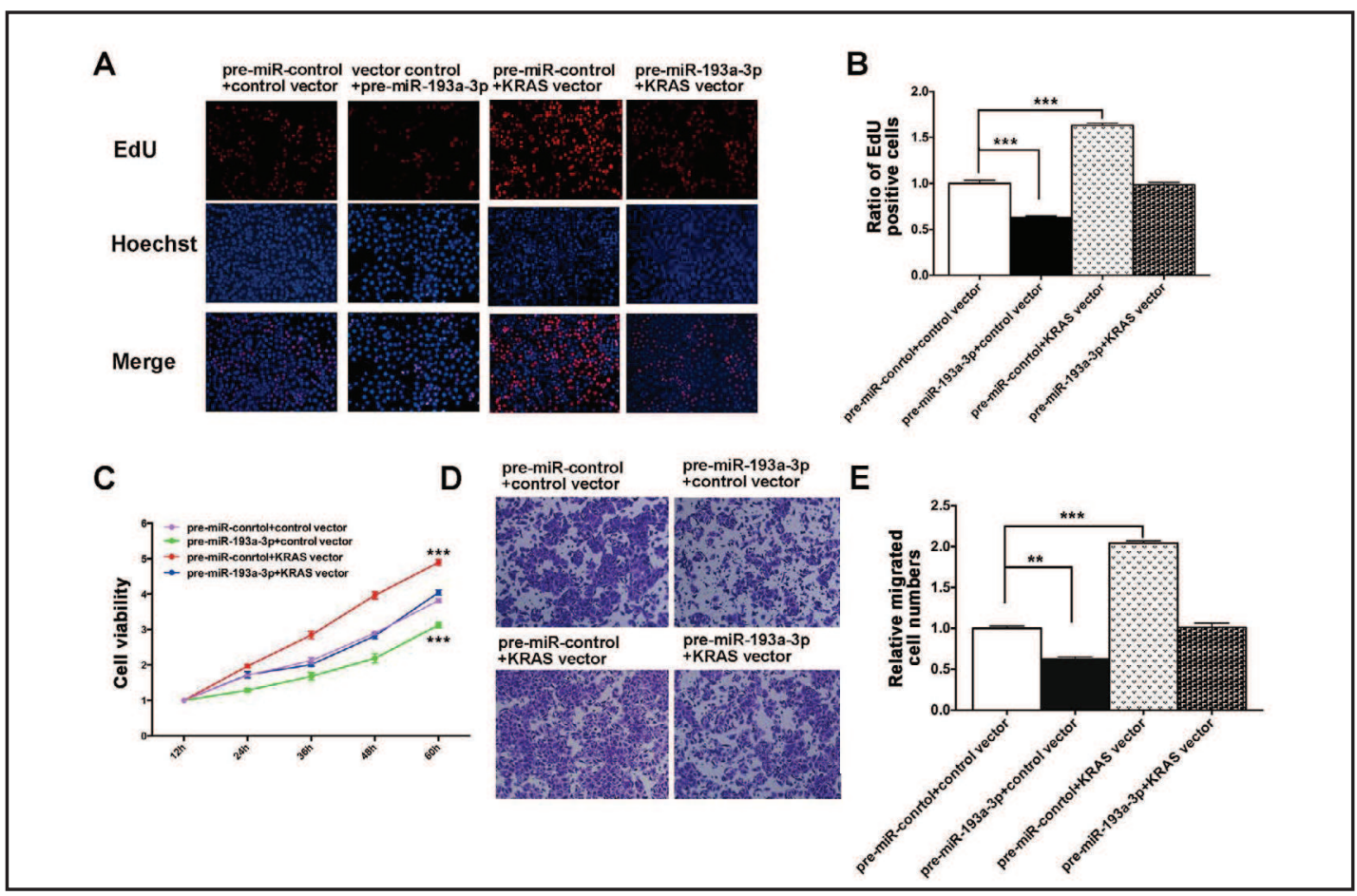

Fig. 7. Co-effect of miR-193a-3p and KRAS in the regulation of viability, proliferation and migration of lung cancer cells. (A and B) EdU assay of the proliferation of A549 cells transfected with equal doses of premiR-control plus control vector, pre-miR-193a-3p plus control vector, pre-miR-control plus KRAS vector, or pre-miR-193a-3p plus KRAS vector; A, representative image; B, quantitative analysis. (C) CCK8 viability assays were performed 12, 24, 36, 48 and $60 \mathrm{~h}$ after the transfection of A549 cells with equal doses of premiR-control plus control vector, pre-miR-193a-3p plus control vector, pre-miR-control plus KRAS vector, or pre-miR-193a-3p plus KRAS vector. (D and E) Transwell analysis of migration of A549 cells treated with equal doses of pre-miR-control plus control vector, pre-miR-193a-3p plus control vector, pre-miR-control plus KRAS vector, or pre-miR-193a-3p plus KRAS vector; D, representative image; E, quantitative analysis. ${ }^{* *} \mathrm{P}<0.01,{ }^{* * *} \mathrm{P}<0.001$.

reporter was unaffected by miR-193a-3p overexpression (Fig. 5E). These findings suggested that the binding sites of KRAS strongly contributed to this miRNA-mRNA interaction. In conclusion, our results demonstrated that miR-193a-3p directly binds to the 3'-UTR of the KRAS mRNA transcript to suppress KRAS expression.

Role of miR-193a-3p in regulating NSCLC cell function by inhibiting KRAS

Subsequently, we investigated whether miR-193a-3p inhibits NSCLC tumourigenesis by silencing KRAS. We assessed the role of KRAS on cell function after overexpression or knockdown of KRAS in A549 cells. The efficiency of KRAS overexpression or knockdown is demonstrated in Fig. 6A-6C. As anticipated, transfection with KRAS siRNA markedly decreased the cell viability compared to the cells transfected with control siRNA, whereas transfection with the KRAS-overexpression plasmid increased cell viability (Fig. 6D). Transfection with KRAS siRNA markedly decreased the percentage of proliferative EdU-positive cells, whereas transfection with the KRAS-overexpression plasmid increased cell proliferation (Fig. 6E and 6F). Additionally, transfection with KRAS siRNA dramatically reduced the number of A549 cells that passed through the Transwell membrane, whereas transfection with the KRAS overexpression plasmid increased the migration rate (Fig. 6G and 6H). Because miR-193a$3 p$ and its target KRAS had opposite expression patterns and biological functions in NSCLC cells, miR-193a-3p may inhibit NSCLC tumourigenesis by silencing KRAS. 


\section{Cellular Physiology Cell Physiol Biochem 2017:44:1311-1324 and Biochemistry Published online: November 29, 2017 www.karger.com/cpb

To investigate whether miR-193a-3p regulation of cell function is mediated through a KRAS-dependent mechanism, we co-transfected A549 cells with pre-miR-193a-3p and the KRAS-overexpression plasmid. After co-transfection with pre-miR-193a-3p and the KRAS-overexpression plasmid, A549 cells exhibited a significantly higher proliferation rate compared with the cells transfected with pre-miR-193a-3p alone (Fig. 7A and 7B), suggesting that miR-193a-3p-resistant KRAS can attenuate the proliferative effect of miR-193a-3p on A549 cells. Similarly, compared with the cells transfected with pre-miR-193a-3p alone, the cells co-transfected with pre-miR-193a-3p and the KRAS-overexpression plasmid attenuated the inhibitory effect of miR-193a-3p on cell viability (Fig. 7C). In addition, co-transfection with pre-miR-193a-3p and the KRAS-overexpression plasmid dramatically attenuated the inhibitory effect of miR-193a-3p on cell migration (Fig. 7D and 7E). Taken together, these results indicate that miR-193a-3p may regulate the proliferation, viability and migration of NSCLC cells in a KRAS-dependent manner.

\section{Discussion}

For the past decade, the study of miRNAs has grown exponentially. Furthermore, it has become clear that aberrant miRNA expression levels are associated with tumour formation, progression and metastasis. miRNA replacement therapy has shown some benefit in cancer treatment. The rationale for miRNA replacement therapy is that miRNAs play a causative role in tumourigenesis of cancer [22]. Due to the significant roles of miRNAs in cancer, they can function as either a tumour suppressor or an oncogene. Accordingly, miRNAs can also serve as either biomarkers or therapeutic targets in cancer treatments [23].

Lung cancer is the major cause of cancer morbidity and mortality worldwide. Chemotherapy is the main treatment for lung cancer, and drug resistance is a major problem that leads to treatment failure. Previous studies have reported non-coding RNAs, including miRNAs, as novel biomarkers and tools for treatment of NSCLC [24]. A single miRNA can target multiple genes, whereas multiple miRNAs can target a single gene. Recently, miRNA regulatory mechanisms that are dysfunctional in lung cancer have been reported [25-27]. To date, some tumour suppressor miRNAs have been confirmed using cultured cancer cells or animal models of cancer [28, 29]. The development of miRNA-targeted therapeutics has become an important anticancer strategy. miR-193a-3p, one of the best-known miRNAs involved in carcinogenesis, is correlated with a wide variety of cellular processes, such as cell differentiation, apoptosis, proliferation, migration and cell-cycle distribution. miR-193a$3 p$ also plays vital roles in several types of human cancers, including lung cancer [30] , and miR-193a-3p was reported to function as a tumour suppressor in lung cancer through the inhibition of ERBB4 translation [31].

In this study, we evaluated the expression patterns of miR-193a-3p in NSCLC tissues and observed that miR-193a-3p could function as a tumour suppressor in NSCLC. The finding that miR-193a-3p is downregulated in NSCLC prompted us to hypothesize that miR-193a-3p might play an important role in NSCLC carcinogenesis. Consistent with this hypothesis, some studies have found that miR-193a-3p can function as a tumour suppressor in lung cancer $[31,32]$. To illustrate this, we used an intratumoural injection of Chol-miR-193a-3p as an "miRNA replacement therapy". As expected, the size of tumours decreased in the Chol-miR193a-3p group. Therefore, we searched for the target genes of miR-193a-3p and identified KRAS as a direct target. The results indicated that overexpression of miR-193a-3p may be involved in the progression of the NSCLC by targeting KRAS in this malignant tumour. In future studies, the clinical implications of the expression of miR-193a-3p in cancer tissues should be evaluated.

The KRAS oncogene is frequently involved in a variety of cellular processes, such as proliferation, differentiation and survival. A recent report confirmed that KRAS mutations are commonly found in a variety tumours, such as lung cancer, colon cancer, pancreatic cancer and ovarian cancer [33]. In addition, a recent study identified miR-193a-3p as a candidate therapeutic to treat cancers exhibiting the Ras-high protein signature [34]. 


\section{Cellular Physiology Cell Physiol Biochem 2017;44:1311-1324 and Biochemistry Published online: November 29, 2017 www.karger.com/cpb \\ Fan et al.: MiR-193a-3p Inhibits KRAS in Lung Cancer}

Activating mutations turn KRAS into one of the most prevalent oncogenes, but an effective drug targeting mutated KRAS has not yet been developed. Since Johnson et al, identified the first tumour-suppressive miRNA that targets and regulates KRAS [8], legions of studies have confirmed that miRNAs can suppress the function of KRAS in various cancers [35]. In this study, we observed that KRAS was consistently overexpressed in NSCLC tissues compared with normal adjacent tissues. We also demonstrated that KRAS functions as an essential pro-proliferation factor in NSCLC; silencing KRAS expression in NSCLC cells through siRNA inhibited cell viability, proliferation and migration, whereas overexpressing KRAS strongly promoted cell viability, proliferation and migration. Interestingly, we identified discordance between KRAS protein and mRNA levels in human NSCLC tissues, suggesting that a posttranscriptional regulatory mechanism is involved in KRAS repression. Because miR-193a-3p is an upstream regulator of KRAS, restoration of miR-193a-3p expression may suppress KRAS in vivo. Further studies are needed to characterise the feasibility of targeting miR-193a-3p in NSCLC therapy and to develop simplified and cost-effective manipulation methods.

Taken together, this study delineated a critical role of miR-193a-3p as a tumour suppressor in NSCLC and found that miR-193a-3p can directly regulate KRAS. Regulation of KRAS by miR-193a-3p may be one of the reasons why the downregulation of miR-193a-3p during NSCLC carcinogenesis promotes tumour growth. In the future, personalized cancer medicine can be realized by designing specific miRNA mimics or antagonists for individuals based on individual patient miRNA expression profiles, and this study may provide a potential novel avenue for future NSCLC therapy.

\section{Disclosure Statement}

No.

\section{References}

1 Rulli E, Marabese M, Torri V, Farina G, Veronese S, Bettini A, Longo F, Moscetti L, Ganzinelli M, Lauricella C, Copreni E, Labianca R, Martelli O, Marsoni S, Broggini M, Garassino MC, trialists T: Value of KRAS as prognostic or predictive marker in NSCLC: results from the TAILOR trial. Ann Oncol 2015;26:2079-2084.

-2 Siegel R, Naishadham D, Jemal A: Cancer statistics, 2012. CA Cancer J Clin 2012;62:10-29.

3 Koscianska E, Krzyzosiak WJ: Current understanding of the role of microRNAs in spinocerebellar ataxias. Cerebellum Ataxias 2014;1:7.

4 Calin GA, Sevignani C, Dumitru CD, Hyslop T, Noch E, Yendamuri S, Shimizu M, Rattan S, Bullrich F, Negrini M, Croce CM: Human microRNA genes are frequently located at fragile sites and genomic regions involved in cancers. Proc Natl Acad Sci U S A 2004;101:2999-3004.

-5 Zhu X, Fu C, Zhang L, Xu G, Wang S: MiRNAs associated polymorphisms in the 3'UTR of MET promote the risk of non-small cell lung cancer. Cell Physiol Biochem 2015;37:1159-1167.

6 Lin L, Tu HB, Wu L, Liu M, Jiang GN: MicroRNA-21 Regulates Non-Small Cell Lung Cancer Cell Invasion and Chemo-Sensitivity through SMAD7. Cell Physiol Biochem 2016;38:2152-2162.

7 Yan A, Yang C, Chen Z, Li C, Cai L: MiR-761 Promotes Progression and Metastasis of Non-Small Cell Lung Cancer by Targeting ING4 and TIMP2. C Cell Physiol Biochem 2015;37:55-66.

-8 Johnson SM, Grosshans H, Shingara J, Byrom M, Jarvis R, Cheng A, Labourier E, Reinert KL, Brown D, Slack FJ: RAS is regulated by the let-7 microRNA family. Cell 2005;120:635-647.

-9 Kasinski AL, Kelnar K, Stahlhut C, Orellana E, Zhao J, Shimer E, Dysart S, Chen X, Bader AG, Slack FJ: A combinatorial microRNA therapeutics approach to suppressing non-small cell lung cancer. Oncogene 2015;34:3547-3555.

10 Deng H, Lv L, Li Y, Zhang C, Meng F, Pu Y, Xiao J, Qian L, Zhao W, Liu Q Zhang D, Wang Y, Zhang H, He Y, Zhu J: The miR-193a-3p regulated PSEN1 gene suppresses the multi-chemoresistance of bladder cancer. Biochim Biophys Acta 2015;1852:520-528.

11 Nakano H, Yamada Y, Miyazawa T, Yoshida T: Gain-of-function microRNA screens identify miR-193a regulating proliferation and apoptosis in epithelial ovarian cancer cells. Int J Oncol 2013;42:1875-1882. 


\section{Cellular Physiology Cell Physiol Biochem 2017;44:1311-1324

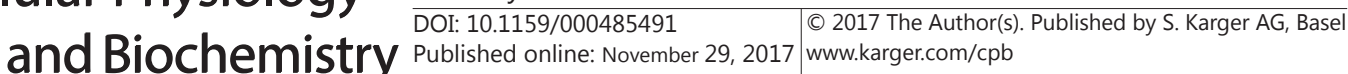 \\ Fan et al.: MiR-193a-3p Inhibits KRAS in Lung Cancer}

12 Tsai KW, Leung CM, Lo YH, Chen TW, Chan WC, Yu SY, Tu YT, Lam HC, Li SC, Ger LP, Liu WS, Chang HT: Arm Selection Preference of MicroRNA-193a Varies in Breast Cancer. Sci Rep 2016;6:28176.

$\checkmark 13$ Chandrasinghe P, Stebbing J, Warusavitarne J: The MACC1-SPON2 axis: A new biomarker and therapeutic target in colorectal cancer. Oncogene 2016;10.1038/onc.2016.322

14 Deng W, Yan M, Yu T, Ge H, Lin H, Li J, Liu Y, Geng Q, Zhu M, Liu L, He X, Yao M: Quantitative proteomic analysis of the metastasis-inhibitory mechanism of miR-193a-3p in non-small cell lung cancer. Cell Physiol Biochem 2015;35:1677-1688.

15 Fan Q, Meng X, Liang H, Zhang H, Liu X, Li L, Li W, Sun W, Zhang H, Zen K, Zhang CY, Zhou Z, Chen X, Ba Y: miR-10a inhibits cell proliferation and promotes cell apoptosis by targeting BCL6 in diffuse large B-cell lymphoma. Protein Cell 2016;7:899-912.

16 Chen X, Guo X, Zhang H, Xiang Y, Chen J, Yin Y, Cai X, Wang K, Wang G, Ba Y, Zhu L, Wang J, Yang R, Zhang Y, Ren Z, Zen K, Zhang J, Zhang CY: Role of miR-143 targeting KRAS in colorectal tumorigenesis. Oncogene 2009;28:1385-1392.

-17 Lewis BP, Shih IH, Jones-Rhoades MW, Bartel DP, Burge CB: Prediction of mammalian microRNA targets. Cell 2003;115:787-798.

18 Krek A, Grun D, Poy MN, Wolf R, Rosenberg L, Epstein EJ, MacMenamin P, da Piedade I, Gunsalus KC, Stoffel M, Rajewsky N: Combinatorial microRNA target predictions. Nat Genet 2005;37:495-500.

19 John B, Enright AJ, Aravin A, Tuschl T, Sander C, Marks DS: Human MicroRNA targets. PLoS Biol 2004;2:e363.

20 Ambros V: The functions of animal microRNAs. Nature 2004;431:350-355.

21 Bartel DP: MicroRNAs: genomics, biogenesis, mechanism, and function. Cell 2004;116:281-297.

22 Ma N, Zhang W, Qiao C, Luo H, Zhang X, Liu D, Zang S, Zhang L, Bai J: The Tumor Suppressive Role of MiRNA509-5p by Targeting FOXM1 in Non-Small Cell Lung Cancer. Cell Physiol Biochem 2016;38:1435-1446.

-23 Inamura K, Ishikawa Y: MicroRNA In Lung Cancer: Novel Biomarkers and Potential Tools for Treatment. J Clin Med 2016;5:

24 Huang C, Ma R, Yue J, Li N, Li Z, Qi D: MiR-497 Suppresses YAP1 and Inhibits Tumor Growth in Non-Small Cell Lung Cancer. Cell Physiol Biochem 2015;37:342-352.

-25 Niu X, Liu S, Jia L, Chen J: Role of MiR-3619-5p in beta-Catenin-Mediated Non-Small Cell Lung Cancer Growth and Invasion. Cell Physiol Biochem 2015;37:1527-1536.

-26 Ma T, Zhao Y, Wei K, Yao G, Pan C, Liu B, Xia Y, He Z, Qi X, Li Z, Wang J, Shao Y: MicroRNA-124 Functions as a Tumor Suppressor by Regulating CDH2 and Epithelial-Mesenchymal Transition in Non-Small Cell Lung Cancer. Cell Physiol Biochem 2016;38:1563-1574.

27 Li J, Yu T, Cao J, Liu L, Liu Y, Kong HW, Zhu MX, Lin HC, Chu DD, Yao M, Yan MX: MicroRNA-148a Suppresses Invasion and Metastasis of Human Non-Small-Cell Lung Cancer. Cell Physiol Biochem 2015;37:1847-1856.

28 He Z, Xia Y, Pan C, Ma T, Liu B, Wang J, Chen L, Chen Y: Up-Regulation of MiR-452 Inhibits Metastasis of NonSmall Cell Lung Cancer by Regulating BMI1 Cell Physiol Biochem 2015;37:387-398.

29 Kopp F, Wagner E, Roidl A: The proto-oncogene KRAS is targeted by miR-200c. Oncotarget 2014;5:185-195.

30 Kwon JE, Kim BY, Kwak SY, Bae IH, Han YH: Ionizing radiation-inducible microRNA miR-193a-3p induces apoptosis by directly targeting Mcl-1. Apoptosis 2013;18:896-909.

-31 Liang H, Liu M, Yan X, Zhou Y, Wang W, Wang X, Fu Z, Wang N, Zhang S, Wang Y, Zen K, Zhang CY, Hou D, Li J, Chen X: miR-193a-3p functions as a tumor suppressor in lung cancer by down-regulating ERBB4 J Biol Chem 2015;290:926-940.

-32 Yu T, Li J, Yan M, Liu L, Lin H, Zhao F, Sun L, Zhang Y, Cui Y, Zhang F, Li J, He X, Yao M: MicroRNA-193a-3p and -5p suppress the metastasis of human non-small-cell lung cancer by downregulating the ERBB4/PIK3R3/ mTOR/S6K2 signaling pathway. Oncogene 2015;34:413-423.

-33 Karandish F, Mallik S: Biomarkers and Targeted Therapy in Pancreatic Cancer. Biomark Cancer 2016;8:2735.

-34 Seviour EG, Sehgal V, Mishra D, Rupaimoole R, Rodriguez-Aguayo C, Lopez-Berestein G, Lee JS, Sood AK, Kim MP, Mills GB, Ram PT: Targeting KRas-dependent tumour growth, circulating tumour cells and metastasis in vivo by clinically significant miR-193a-3p. Oncogene 2017;36:1339-1350.

35 Deng M, Tang H, Zhou Y, Zhou M, Xiong W, Zheng Y, Ye Q, Zeng X, Liao Q, Guo X, Li X, Ma J, Li G: miR216b suppresses tumor growth and invasion by targeting KRAS in nasopharyngeal carcinoma. J Cell Sci 2011;124:2997-3005. 